\title{
An Upcycling Tokenization Method for Credit Card Numbers
}

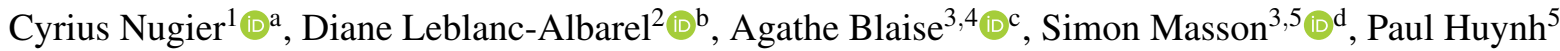 \\ af and Yris Brice Wandji Piugie $\mathrm{C}^{6,7}$ 甫 \\ ${ }^{1}$ LAAS-CNRS, Université de Toulouse, Toulouse, France \\ ${ }^{2}$ CNRS, IRISA, INSA de Rennes, Rennes, France \\ ${ }^{3}$ Thales, Gennevilliers, France \\ ${ }^{4}$ Sorbonne Université, Paris, France \\ ${ }^{5}$ Université de Lorraine, INRIA, Loria, CNRS, Nancy, France \\ ${ }^{6}$ FIME EMEA, Caen, France \\ ${ }^{7}$ Normandie Université, UNICAEN, ENSICAEN, CNRS, GREYC, 14000 Caen, France \\ cyrius.nugier@laas.fr, diane.leblanc-albarel@irisa.fr,agathe.blaise@thalesgroup.com, simon.masson@loria.fr, \\ paul.huynh@loria.fr, brice.wandji@fime.com
}

Keywords: $\quad$ Token Service Provider, Credit Card Numbers

Abstract: Internet users are increasingly concerned about their privacy and are looking for ways to protect their data. Additionally, they may rightly fear that companies extract information about them from their online behavior. The so-called tokenization process allows for the use of trusted third-party managed temporary identities, from which no personal data about the user can be inferred. We consider in this paper tokenization systems allowing a customer to hide their credit card number from a webshop. We present here a method for managing tokens in RAM using a table. We refer to our approach as upcycling as it allows for regenerating used tokens by maintaining a table of currently valid tokens. We compare our approach to existing ones and analyze its security. Contrary to the main existing system (Voltage), our table does not increase in size nor slow down over time. The approach we propose satisfies the common specifications of the domain. It is validated by measurements from an implementation. By reaching 70 thousand tries per timeframe, we almost exhaust the possibilities of the "8-digit model" for properly dimensioned systems.

\section{INTRODUCTION}

Internet users leave digital fingerprints behind them, even behind a pseudonym. All this data can be studied in order to infer information about the users and their behaviors. This is notably done on the largest e-commerce platforms and social networks. More specifically, buying patterns of consumers are extremely valuable to companies, as they help them understand their market. In addition, the storing of online payment data is not secure and there is always a risk of a data leak. Last years have witnessed numerous episodes of credit cards thefts online, like

\footnotetext{
a (iD https://orcid.org/0000-0003-1276-0296

b (iD https://orcid.org/0000-0001-5979-8457

c (iD https://orcid.org/0000-0002-9598-8482

d(iD https://orcid.org/0000-0001-8778-2575

e (iD) https://orcid.org/0000-0002-6965-3427

f(D) https://orcid.org/0000-0002-6258-6073
}

the Davinci breach (Krebs, Brian, 2019) in February 2019 (2.15 M stolen credit cards), the BigbadaboomII (Thales group, 2018) in March 2018 where compromised details were released by the FIN7 threat group, and the Bigbadaboom-III (Sussman, 2020) in January 2020 (30 M stolen credit cards), to name only a few. It is therefore natural for customers to worry about their card number being leaked and to expect the damage to be mitigated in case of leakage.

According to (Whatman, 2020), there were 2.8 billion credit cards worldwide in 2019, and the number of credit card transactions in 2017 was estimated to 41 billion in the US. Also, according to the Census Bureau of the Department of Commerce (U.S. Department of Commerce, 2020), the estimate of U.S. retail e-commerce sales for the second quarter of 2020 was $\$ 211.5$ billion, an increase of 31.8 percent $( \pm 1.2 \%)$ from the first quarter of 2020 . In total, the estimated e-commerce sales represent $17 \%$ of all 
sales.

The ability to use multiple fake but verifiable credit card numbers over time and to limit their spending capacity allows for the protection of the customer's identity and data. However, assigning these new bank identities called "tokens" to customers in an efficient and secure way is still a challenge.

Our Contributions: In this paper, we study CCNs tokenization systems. We take a look at existing approaches to create a tokenization system and then propose one that avoids their main issues while still complying with the domain specifications. We refer to our approach as upcycling since it allows for regenerating used tokens by maintaining a table of currently valid tokens, thus eliminating the need to create new tables over time and slowing down calculations. To satisfy auditability requirements, transactional data will be stored in an external database. We propose a proof of concept implementation and study its memory and time performances.

Organization of the Paper Section 2 introduces the background of tokenization systems for CCNs. Section 3 contains related works on the domain. Section 4 presents our approach and the different algorithms composing it. We study the compliance with the specifications and the impact of different parameters on our system. Section 5 gives experimentation and benchmark results of our proof-of-concept implementation. The TSP source code used for the tests and evaluation is available on git as supplementary material (Albarel., 2021) Section6 draws conclusions from our work.

\section{BACKGROUND}

In this section we introduce the domain of tokenization systems for credit card numbers.

\subsection{Credit Card Numbers}

Credit card numbers (CCNs), or primary account numbers (PANs), consist of a maximum of 19 digits that identify the card issuer and the cardholder. As depicted in Figure 1, they are composed up of three main elements, in accordance with ISO/IEC 7812-1 (International Organization for Standardization, 2017):

1. The issuer identification number (INN), which corresponds to the leading 8 numerical digits.

2. The individual account number (IAN), which can be of variable length - between 1 and 10 digits.

3. A check digit (CD) computed from all the preceding digits of the PAN using the Luhn algo- rithm (Luhn, 1960).

The individual account number is usually 7-digit long, which amounts to a total of 16 digits in a PAN.

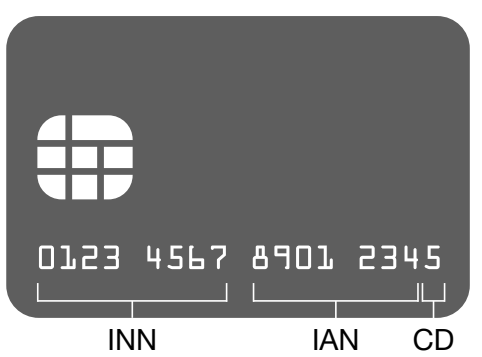

Figure 1: Credit Card Numbers format.

As for the payment token, it is possible to adopt a structure that slightly deviates from the conventional format (Payment Card Industry, 2015). For instance, the first four digits can be used to identify the card issuer; the last four digits are fixed and can be used for integrity or error detection purposes in the token (such as a checksum); the remaining 8 "open" digits in the middle identify the token. In the remaining sections of the paper, this format - shown in Figure 2- will be considered.

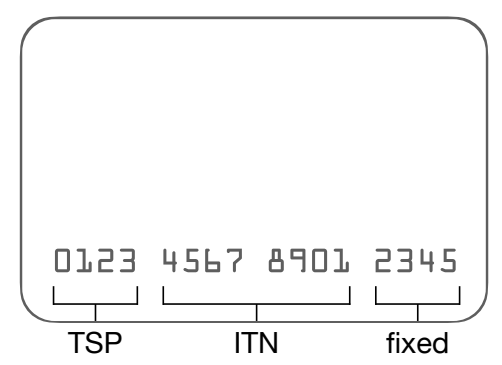

Figure 2: Possible token format.

At the back of a physical card, three additional digits form the Card Verification Value (CVV). Their purpose is to guarantee the physical possession of the card and therefore, they have no use in a digital application like tokens. Moreover, according to PCI compliance rules, the CVV should never be stored except by the card issuer (Payment Card Industry, 2020).

\subsection{Tokenization}

Nowadays, in order to increase the security of online transactions, many payment systems use to provide users with a temporary identity. This reduces traceability, limits the possible inferences and risks of data leakage.

A common approach is tokenization: this process replaces an existing payment card number with a substitute value called token, that is issued by a trusted 


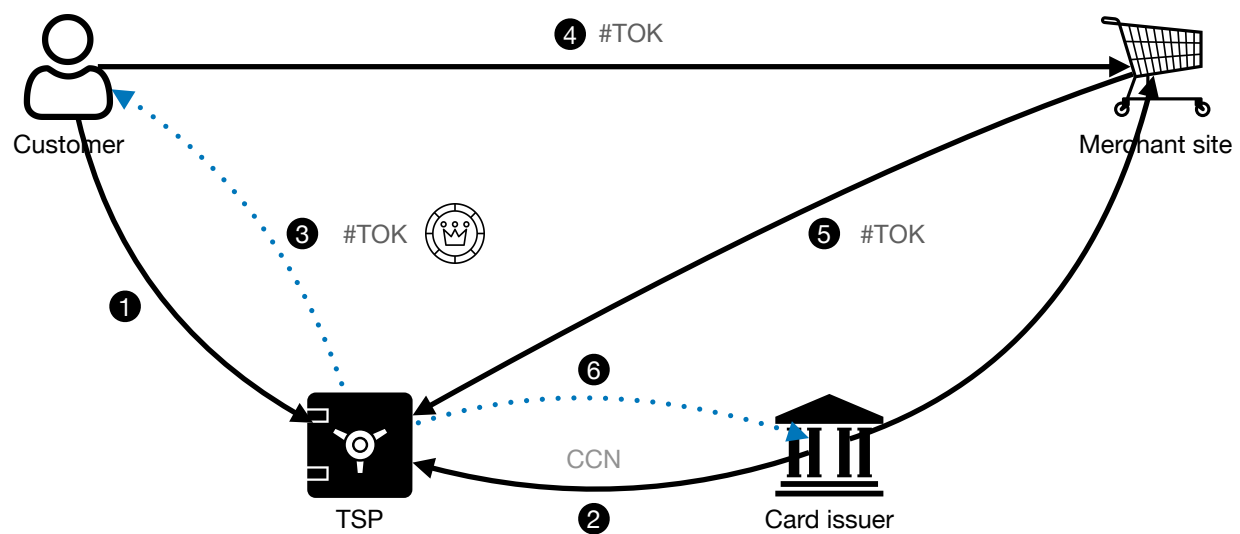

(1) Token request

(2) Data retrieval

(3) Token emission

(4) Payment attempt

(5) Payment request

6 Detokenization

(7) Payment

Figure 3: A Standard Tokenization System.

third-party, called a Token Service Provider (TSP), that serves as a proxy masking the user's real identity. A token is then used during a payment transaction, allowing one to proceed with the payment without exposing actual bank details. The Token Service Provider associates the original card number with the tokens and stores all sensible data securely.

More precisely, the TSP manages the entire lifecycle of tokens. The typical scenario in this context is depicted in Figure 3 .

1. Token Request. The customer requests a token from the TSP.

2. Query. The TSP queries all data needed for the creation of the token (usually from the card issuer or customer).

3. Tokenization. The TSP creates a token from the Credit Card Number and sends it to the customer.

4. Purchase. The customer purchases an item or service from an online shop and transmits the token number instead of their Credit Card Number.

5. Payment Request. The merchant site returns the token to the TSP and claims the payment.

6. Detokenization. The TSP converts the token back to the correct Credit Card Number and transmits the payment request to the card issuer

7. Payment. The card issuer satisfies the payment request from the merchant site.

The main role of the TSP is the role of the token vault: establishing a correspondence between the token and the Credit Card Number. The TSP can endorse additional responsibilities like domain management (giving additional security by limiting the tokens to specific channels or retail domains) and authentication (ensuring during the detokenization process that the token has been legitimately used by the correct client). For this, it can verify the identity of the users, by asking them to claim their identity with a password, through multi-factor authentication, or with a cryptographic signature, e.g., the ECDSA standard (Johnson et al., 2001).

Card issuers can endorse the role of TSP, allowing full control of the tokenization process. Otherwise, card issuers may use a third-party TSP and integrate it with their payment systems.

\subsection{Specifications}

The specifications for the tokenization systems are listed hereafter. They ensure that the goals of protection of the customer are met without loss of quality of service.

1. Unicity. Each token should be attributed to at most one user at any given time.

2. Expiry. A token has a maximum number of uses, a maximum spending amount and/or an expiry date.

3. Formatting. The format of the token should be identical to CCNs.

4. Distribution. The distribution of the tokens' open digits should be uniform.

5. Unlinkability. Tokens should not be linkable to one another, or to a user.

6. Timeframe. Tokenization and Detokenization computation times should not exceed a given timeframe value denoted $T f$. In this paper, we consider $T f$ to be $100 \mathrm{~ms}$ as a constraint from card issuers so the TSP slowdown is not too noticeable from the customer point of view.

7. Unforgeability. An adversary should be unable to forge a valid token and obtain payment.

8. Reusability. The space of all tokens' open digits being smaller than the expected number of token 
requests, the same open digits should be able to be issued several times.

9. Auditability. This depends on the legislation the TSP is submitted to. It varies from one country to another. We consider that the time and data for all tokenizations and all detokenizations (succeeded or failed) should be stored for 5 years.

10. Security. Any storage of data should be as secure as possible as long as all previous specifications are validated.

11. Limited Storage Space. Any data storage used should be small enough to not create additional costs for TSPs, as long as all previous specifications are validated.

\section{RELATED WORK}

In this section, we present the related work on formatpreserving encryption (FPE) and static pre-computed tables. We position our contribution with respect to the described related work.

The authors in (Díaz-Santiago et al., 2014) formally define tokenization systems and identify three different systems that solve the problem of tokenization both securely and efficiently. The first one uses format-preserving encryption (FPE), while the latter two systems rely on off-the-shelf cryptographic primitives using ordinary block ciphers, stream ciphers supporting initialization vectors, and physical random number generators. The difference between both relies on whether pairs of token and PAN are stored in the card-vault in the encrypted form or not. The authors also give several security notions and provably secure constructions. However, they do not consider adaptive constructions, and unlike (Cachin et al., 2017), they do not address updatable tokens. The authors also refer to the "Voltage Security" solution (Voltage Security, 2012) as the only solution at this time to the tokenization problem with known cryptographic guarantees, using static pre-computed tables.

As a matter of fact, most existing solutions are static and do not provide key updatability, i.e., they do not regularly update the cryptographic keys, while maintaining the tokens' consistency, which could lead to security issues. Therefore, in most practical deployments, cryptographic keys must be re-keyed periodically to ensure continued security. (Cachin et al., 2017) constructs two tokenization models for updatable tokenization with key evolution, in which a key exposure does not disclose relations among tokenized data in the past, and where the updates to the tok- enized data set can be made by an untrusted entity and preserve the consistency of the data. The authors formally define the security requirements that guarantee unlinkability among different tokens generated from a same user.

\subsection{Format-preserving Encryption}

One common option for the generation of tokens is the use of Format-preserving Encryption (FPE) (Bellare et al., 2009). FPE can be seen as a key-indexed pseudorandom permutation of the set of all values of correct format, called domain. The keyspace can be much greater than the domain (should have cryptographically big enough size).

FPE has gradually emerged as a useful tool in applied cryptography in recent years. The initial motivation for its use came from the encryption issues raised by companies looking for specific data formats. For instance, encrypting distinct and unpredictable 16-digit credit card numbers with traditional block ciphers would expand the data and change its format, which would require modifications of the applications or databases at huge expense (Liu et al., 2010).

The goal of FPE is to avoid the need for a database to link a token to a CCN. The natural use of FPE is to encrypt the individual account digits (and the checksum digit) as the 8 open digits of the token. The validation of the token is then done by decryption of the 8 open digits of the token to retrieve a card number that is then transmitted to the bank for payment. If the token given by the merchant to the TSP is incorrect, the corresponding bank account would be invalid.

For now, the domain size remains out of reach of known attacks on small domains, as it is big enough. For example, in (Hoang et al., 2018), attacks are provided on domains up to 8 bits. Following the attacks discovered in (Durak and Vaudenay, 2017), the National Institute of Standards and Technology (NIST) recommends using domains of at least one million elements (NIST, 2020). With one hundred million domains, the CCNS FPE systems are still out of reach for now, but this should be a concern for a long-lasting system.

The first limitation found in the use of FPE is that the map from users to the 8 open digits is not bijective, since two banks with different fixed digits can issue the same open digits to two different users, e.g., John Doe, client ID 12345678 at BankA and Michel Dupont, client ID 12345678 at BankB. Such a scenario would imply that the tokens generated by these users would always be the same. These two users cannot have tokens issued with the same key. Another possibility is to have an injective map from card is- 
suers to TSP that would avoid this type of conflict, i.e., having a single TSP per card issuer.

Assuming that the indexing secret keys are changed regularly, two different card numbers with two different keys can yield the same token. In this case, in the verification phase, it would be impossible to differentiate the two tokens (see Specification 1 of Section 2.3) except with the inclusion of additional identification information that would need to be stored. Additionally, the pairs (token, secret key) would anyway be kept in a database in order to know which key needs to be used to decipher a given token. Storing these pairs and identification data defeats the advantage of using FPE instead of having a static precomputed table (knowingly reducing storage space).

Besides, if we keep the same secret key across time, it opens the possibility for attackers to trace a token number, since it is permanently linked to the card number. This would not comply with Specification 5 of Section 2.3

To summarize, the use of FPE would either create collisions or require a database. In the latter case, it just creates an overhead that can be avoided with a classical table/database. In conclusion, our system will not use FPE.

\subsection{Static Pre-computed Tables}

We call static pre-computed tables those which contains all possible token values computed in advance.

Voltage Security proposed a way to generate and use tokens (Voltage Security, 2012) in 2012, according to the Payment Card Industry Data Security Standards (PCI DSS) requirements (Payment Card Industry, 2020), which include for example the need to install and maintain a firewall configuration to protect cardholder data, to protect the stored data, to encrypt every transmission of cardholder data across public networks, etc.

During tokenization, a token is randomly associated with the card number. With a good random number generator and good management of the table where the tokens are stored, this solution is completely in accordance with the PCI DSS and allows for a quick tokenization process. During the detokenization phase, it simply checks if the token exists in the table and, in that case, the associated bank account is returned.

However, the Voltage approach leaves some doubts about the security of the table, which is kept in plain text. More importantly, no mechanism avoids the saturation of the table. This could be a problem if the maximum number of $10^{8}$ tokens is reached. For certain this technique allows for a tokenization that is fast on average, but problems arise whenever the number of tokens increases. What Voltage proposes to tackle this issue is to create a new table when the previous one is saturated. However, this mechanism increases the detokenization times since multiple tables have to be searched to find the correct bank account. Additionally, this creates the need for more data storage space.

The main design consideration of our contribution was to remove the increase of storage and computations over time. Although (Cachin et al., 2017) does a first step by providing encryption and key updatability, it does not allow for table cleaning and token reusability. Up to our knowledge, we are the first to provide all of these features. Our proposition allows one to have a dynamic table that is more portable. Moreover, we propose a method that regularly cleans the table, considering an expiry time for tokens and a maximum number of uses. This way, our system allows reusing the tokens that are no longer in use in order to ensure the sustainability of our system over a long time.

\section{OUR UPCYCLING TOKENIZATION METHOD}

In this section, we introduce our upcycling tokenization method. We discuss how to properly chose parameters and introduce the composing functions of our system.

\subsection{System Overview}

Our approach is a modification of Voltage's static precomputed table that integrates a cleaning mechanism. Thanks to this mechanism, it is not necessary to create an additional table every time the previous one starts to be too full. Additionally, we encrypt the table for more security, and we include a mechanism for updating encryption keys, as well as a mechanism to detect accidental modifications in the table. We include an extra database for audit purposes.

This construction has been built on the supposition that the storage of all cryptographic secrets (keys, initialization values) is proper and secure. Also, we assume the randomness generators to be cryptographically strong.

The basis of our approach is the creation of a table in RAM indexed by the token numbers, which therefore consists of $n_{\max }$ rows, where $n_{\max }$ is the number of possible tokens, i.e., $10^{8}$ in the 8-digit model. To retrieve data from a token to complete a payment, there is just to retrieve the data contained in the row of 
the table corresponding to the token. A row is therefore composed of:

- The credit card number CN, stored on 64 bits to include the $16 \mathrm{CCNS}$ digits

- A timestamp expiry = current time + lifespan, which is expressed in seconds and thus stored over 32 bits, or expressed with a larger range or higher precision and stored over 64 bits. It indicates the expiry date of the token.

- A counter num_uses of the remaining uses of the token. An 8-bit integer is enough for the predicted use of the tokens since increasing the number of uses of the same token increases its traceability. If num_uses $=1$ for all tokens, this field can be removed.

- A counter credit of the remaining possible spending with this token. This field is also optional.

- If the tokenization scheme includes an authentication mechanism, all the required data should be stored in the row, e.g., password, verification keys, email address, or phone number for multi-factor authentication.

- The random number rand used to generate the token (32 bits) can also be stored, it allows for the verification of the row during the Clean_table operation.

We propose the functions Tokenization and Detokenization to create and use tokens, and also a Clean_table function verifies the contents of the table and removes decayed tokens.

Ideally, the table should be created contiguous in memory so that the access to the $n^{\text {th }}$ element could be done by calculating the offset from the first element. This way, tokens are perfectly indexed by their value. This allows keeping a constant access time for lookup while keeping a minimal database space (Specification 6). For security purposes, it is also recommended that the database should be encrypted via a secret key.

Note that according to the volume of data to store for authentication the table size can vary. However, a good computer could have it entirely in RAM without the use of non-volatile memory.

The number of uses given for a token and its lifespan should be very carefully chosen according to the expected rate of use of tokens. For example, imagine an e-commerce scenario, we can say that tokens have a 10-minute lifespan, since most transactions that are longer than this become invalid for security reasons.

If the tokens have only one use, every consumer that fails to complete the transaction leaves an unused token in the table. If they have more than one, it is increasingly probable that at least one will never be used in a transaction and leave a token in the table. The expiry of the tokens allows to detect them and remove them periodically.

The best parameters would be to choose the minimal numUses and lifespan values that validate the design constraints on the system. The cleaning of the table (Clean_table) should be executed periodically, e.g., once a day or week or month, according to the expected number of tokens that will not be used during their lifespan, the period should be high enough to ensure there is always room to create new tokens.

All the actions done in the table and every call to our functions can be stored with a timestamp in a permanent external encrypted database to comply with auditability requirements. According to the legislation applied on the TSP, some fields are expected to be stored or not. The legislation may require the data to be stored for a given amount of time and deleted afterward. Since this is classical database management, we will just present when our system adds data into the database.

\subsection{Description of the Functions}

The three following functions enable one to complete the whole tokenization and detokenization process, as well as the maintenance of the table. Hereafter follows a detailed description of each process.

\subsubsection{Tokenization}

The tokenization process consists in generating a token tok from a card number CN. It also implies storing the data concerning the user in order to proceed with subsequent detokenizations.

The algorithm Tokenization(Tab, CN, num_uses, credit expiry, auth, sk) takes as input the table Tab, the credit card number of the user CN, the maximum number of uses of the token num_uses, a timestamp expiry, and any extra information auth that would be useful to retrieve at the time of detokenization (e.g. cryptographic public key), as well as the system's cryptographic key (and iv) sk.

First, if required, a tokenization call is added to the external database.

Then, the algorithm picks uniformly a 32 bit rand and computes the 32 last bits of

$$
\text { hash }_{32}=\mathrm{SHA}_{224}(\mathrm{CN}, \text { expiry, auth, rand }) \text {. }
$$

To ensure the uniformity of the output distribution, this process is repeated with a new rand while hash $_{32}$ is greater than the biggest multiple of $10^{8}$ written in 32 bits (happens $0.1 \%$ of time). 


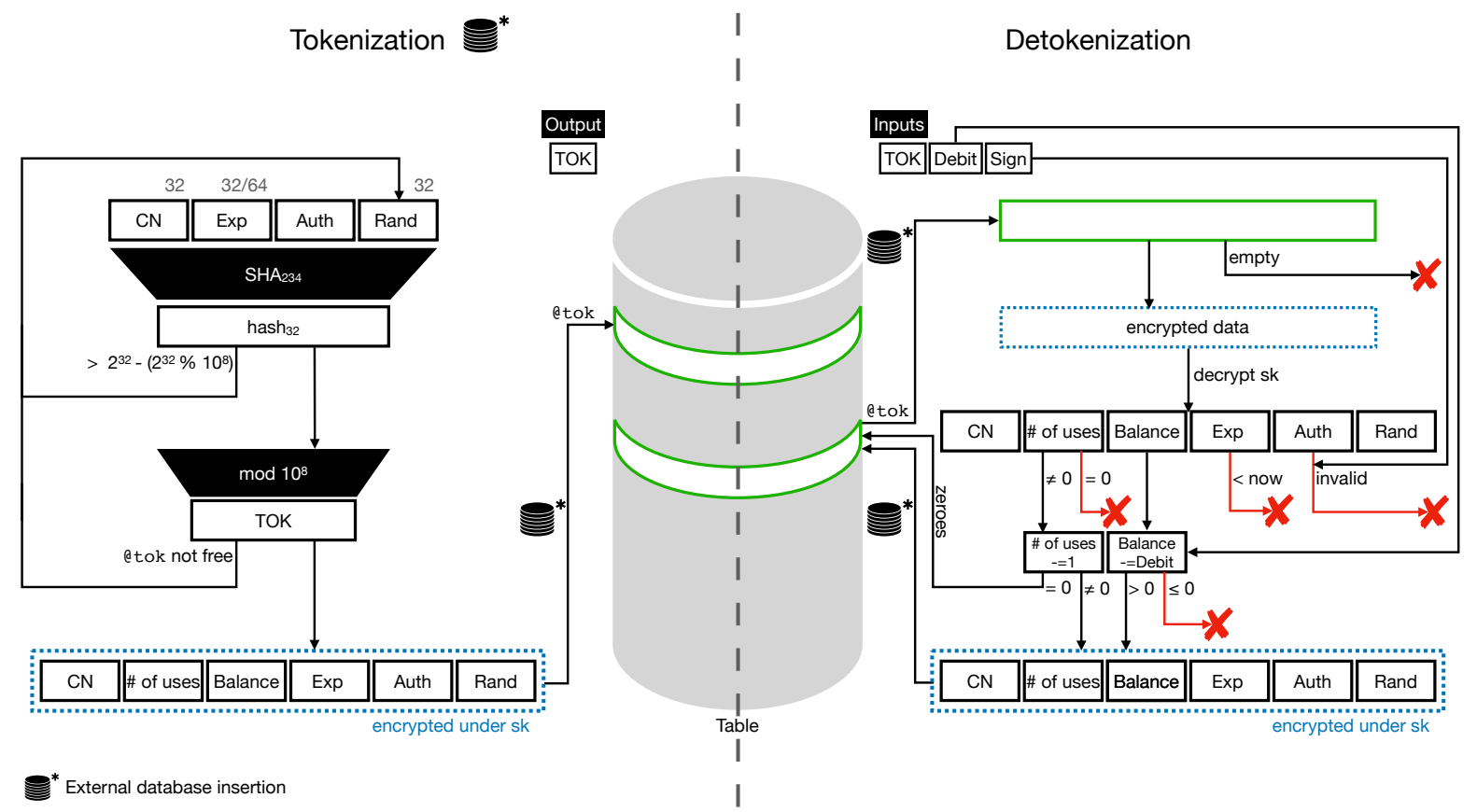

Figure 4: Upcycling Tokenization Table Algorithms.

Then, given $n_{\max }$ the maximum number of rows it computes tok $=$ hash $_{32} \bmod n_{\max }$.

This way, a token (8 open digits) is generated uniformly by hashing the data including rand and reducing the result into the token space by carrying out a modulo $n_{\max }$ operation on it.

num_uses is not included since it will vary over time. Then, it checks whether tok corresponds to an empty row of the table. If the token already exists and is valid, then the process restarts and tries with a fresh rand value until a new token is found. Once an empty row is found, it inserts Encrypt $_{\text {sk }}(\mathrm{CN} \mid$ num_uses $\mid$ credit $\mid$ expiry $\mid$ rand $\mid$ auth $)$ in the row.

If the duration of the algorithm came to exceed timeframe, the algorithm would stop with a failure flag. Else, it would return a success flag and the 8digit token that got created.

The TSP should ensure to format properly the token back to a full token format by adding the TSP identifier and the checksums.

All fields required for audit purposes (e.g., the token number) are transmitted to the external database.

\subsubsection{Detokenization}

The detokenization process consists in retrieving a credit card number $\mathrm{CN}$ from a token tok.

If needed (e.g., to detect malicious users requesting payments), the algorithm saves the detokenization call in the external database.

The algorithm Detokenization(Tab, tok, debit, verif, sk) takes as input the table Tab, the token tok to verify (as an 8-digit value), verif the data possibly required for authentification (e.g., cryptographic signature) and sk the system's cryptographic key (and iv).

It checks whether the table row indexed by tok is empty or not. If it is empty, the token is invalid and the algorithm stops with a failure flag. Otherwise, the row is deciphered with sk to retrieve (CN $\mid$ num_uses $\mid$ expiry $\mid$ credit $\mid$ rand $\mid$ auth). If the token is depleted or invalid, or credit $<$ debit the row is deleted and the algorithm stops with a failure flag. Else, the algorithm Verify_signature(auth, verif) to authenticate the consumer. If Verify_signature returns a failure flag, Detokenization stops and returns a failure flag. We further detail authentication methods in Section 4.5 .

Else, everything went well, num_uses is decremented, debit is substracted from credit. If it reaches 0 , the row is deleted, else the row is kept encrypted in the table. Either way, the $\mathrm{CCN}$ is returned. It can be sent to the card issuer for completion of the payment.

Also, any changes to the token can be reported in the external database for audit purposes. 


\subsubsection{Cleaning the Table}

To perform the cleaning, each row of the table is checked and kept only if the token is still alive and correct.

The algorithm Clean_table (Tab,sk) takes as input the table Tab and sk the system's cryptographic key (and iv). For all rows, it deciphers the row to retrieve (CN $\mid$ num_uses $\mid$ credit $\mid$ expiry $\mid$ rand $\mid$ auth).

The call to this function can be saved in the external table if needed.

The number of remaining uses and the expiry date are verified. If the validity date has passed or if the maximum number of times the token has been used is reached, then the row is erased. In order to detect errors in the table, the algorithm can also compute if $\mathrm{CN}$ is a valid $\mathrm{CCN}$ by verifying its checksum, and whether $\mathrm{SHA}_{224}\left(\mathrm{CN}\right.$, expiry, auth, rand) $\bmod n_{\max }$ is the index of the row. If no error is found, the token stays as it was and the algorithm continues with the next one. Each cleaned row can also be stored in the external database.

Also, keeping the same secret key for a long time is insecure, it should be changed regularly. Clean_table could also be used as a key updater by taking as additional input the new_key. Each row is deciphered with sk, and after normal operations, the rows are inserted back in the table encrypted with new_key, which now acts as sk.

\subsection{Conformity with Specifications}

We prove that given a correct set of parameters, each specification is validated by our approach.

1. Unicity. Each token is unique because Tokenization only returns a non-existing token.

2. Expiry. This is guaranteed by the verification process during Detokenization and the fact that num_uses is decremented after each use. expiry and num_uses are never incremented.

3. Formatting. The token format matches the one of CCNs because of the formatting step at the end of Tokenization.

4. Distribution. Any newly issued token has a number taken from a uniform distribution (property of the hashing function).

5. Unlinkability. Since our systems includes random values into the hash, there is no information deductible from the observation of multiple tokens, even with all other fields of the row being the same. Therefore no link can be inferred between tokens and consumers.
6. Timeframe. Tokenization times can be guaranteed to be bounded, with the probability of failure to deliver a new token being arbitrarily low. However, the implementation optimization, the computer performances, and the value of $T f$ have an impact that we quantified in the upcoming paragraph. Detokenization times are bounded because the table access is in constant time.

7. Unforgeability. Without authentication of the customer during a payment request, a merchant site could submit a random illegitimate token and send a payment request. The probability of success is equal to the number of currently valid tokens divided by the total number of tokens. In a token space of $10^{8}$, this causes a lot of design constraints. Therefore, authentication is needed to ensure this property.

8. Reusability. A properly sized system ensures by cleaning regularly the table that all expired tokens can be used again.

9. Auditability. All functions called and all tokens created and modified can be stored in a permanent external encrypted database according to TSP regulations.

10. Security. The table and the external database are encrypted. Good key management is necessary.

11. Limited Storage Space. For normally sized authentication data, the table can be in a computer's RAM. The external database should contain only the required data to limit costs.

\subsection{Probability of Tokenization Failure}

Let us consider a token space of size $n_{\max }$, the number of already generated tokens $n$ and the number of tries $T$ to generate a new token that can be done in the given timeframe $T f$.

We study the maximum $n$, such that the probability of a failure to create a new token is smaller than $2^{-\lambda}$, where $\lambda$ is the security parameter. The probability of failure is the probability of obtaining consecutively $T$ already existing tokens, which happens with a probability of $\frac{n}{n_{\max }}$. Thus we obtain the following inequality:

$$
\left(\frac{n}{n_{\max }}\right)^{T}<\frac{1}{2^{\lambda}} \Longleftrightarrow n<2^{\log _{2}\left(n_{\max }\right)-\frac{\lambda}{T}} .
$$

When $\lambda \ll T, n \approx n_{\max }$. Therefore with reasonable security parameters, e.g., 128 or 256 , we need to optimize enough the implementation so that $T$ is big enough. As long as this is the case, $n \approx n_{\max }$, and any failure to create a token in an upcycling table is proof 
that either the system isn't properly dimensioned or that the 8-digit model is no longer big enough. (It would imply that around $\approx 100 M$ users are instantaneously in a transaction.)

\subsection{Authentication methods}

Here we propose three authentication solutions to ensure unforgeability.

1. Password: During the token request, the customer chooses a password and sends a hash to the TSP as auth. During detokenization the customer submits verif, Verify_signature will just check whether auth = verif (Halevi and Krawczyk, 1999). Note that using always the same password would allow an observer to infer that multiple detokenizations may be linked.

2. Signature: During the token request, the customer sends a cryptographic public key auth. During detokenization the customer submits verif, its cryptographic signature. Verify_signature is the cryptographic verification of the signature (Johnson et al., 2001). Note that no information can be inferred from proper cryptographic signatures.

3. Multi-factor Authentication: During the token request, the customer sends contact data auth, such as email address or phone number. During detokenization Verify_signature picks a code $C$, sends it via email or SMS to the customer. If the customer then submits verif $=C$, then the authentication is validated (N. Owen and Shoemaker, 2008). Since $C$ is chosen at random independently of the user, no link can be inferred between two detokenizations.

\section{PERFORMANCES}

In this section, we evaluate our solution in terms of table fill rate and time taken by tokenization, detokenization, and the cleaning of the table. We also provide the RAM usage needed to run our solution.

We decided to use the $\mathrm{C}$ language to have good memory management. This choice allows us not only to reduce the amount of RAM used, but also to reduce the token generation time by controlling precisely the size of the data. For the sake of reproducibility, our source code is publicly available on a repository (Albarel., 2021).

\subsection{Tokenization time and Table fill rate}

We performed our experiments on an AMD EPYC 7742 64-Core Processor. Each processor has an average speed of $3240.029 \mathrm{MHz}$.

For each experiment, we generated tokens corresponding to random credit card numbers and then filled our $10^{8}$ rows table with tokens. We tried to generate new tokens until the table is so full that a new token could not be generated in less than the $T_{f}$ timeframe of $100 \mathrm{~ms}$. We repeated this experiment 10 times to obtain statistically significant results. We then evaluate several metrics: the table fill rate before the first failure happened, the number of missed tries that lead to this first failure, the maximum number of tries before inserting a correct value, and the time needed to fill the table before an insertion fails. Hereafter, Figure 5 shows the box plot of these four metrics computed for 10 fill table operations.

Our results show that the table can be filled to a median of $99.99 \%$ with a standard derivation of 0.002 (Figure 5a) before the first failed token creation, which is very satisfying.

The number of tries per timeframe can be bounded between the number of tries before the first failure (Figure 5b) for which the median is 70,268 , with a small standard deviation equal to 634 and the number of tries per timeframe is the number of tries before inserting, which median is equal to 65,251 .

The median time to fill the table before a failure occurs (Figure 5d) is 1344 seconds (i.e., 22 minutes and 44 seconds), also with a very small standard deviation equal to 28 seconds.

Suppose a TSP wants to ensure a very low probability of failure e.g $\frac{1}{2128}$. From 1 , with $T=70000$, we find a maximum table fill rate of $99.8733 \%$, which indicates that the bottleneck is more the size of the table than the performances.

Our experimentation show that single try for tokenization lasts approximately $100 \mathrm{~ms} / 70,268=$ $0.0014233 \mathrm{~ms}$. Since $99.99 \%$ of $10^{8}$ tokens are created in $1350 \mathrm{~s}$, an insertion took in average $0.0135 \mathrm{~ms}$, or 75,750 tokens created per second.

To illustrate, credit cards can do 5000 transactions per second. There is an average of 1 billion credit card transactions per day worldwide, or an equivalent of 11574 transactions per second (CardRates, 2020). Our construction covers 6.5 times this rate. Furthermore, with a 10-minute token lifespan, with maximum token creation speed, a maximum of 45 million valid tokens can be in the table at any given time. So with Clean_table called every 10 minutes, there is no possibility for the table fill rate to go over the security threshold. 

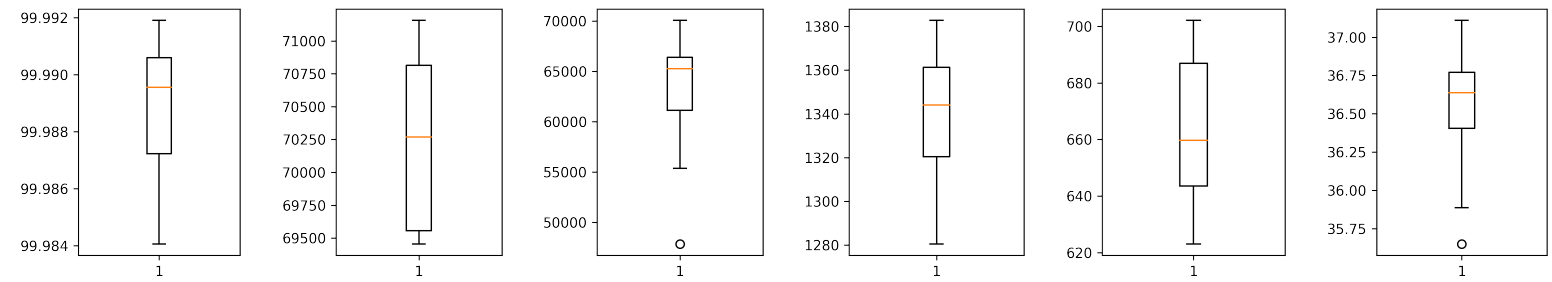

(a) Final table fill (b) Tries before firs rate $(\%)$ (c) Max number of (d) Time to fill the tries before success table (s) (e) Time to detok- (f) Time to clean the enize the table (s) table (s) Figure 5: Box plot of evaluation metrics for Tokenization, Detokenization and Clean_table.

\subsection{Detokenization and Cleaning table time}

Finally, we also measured the average time needed to detokenize and clean the table. In addition to the tokenization metrics, Figure 5 also provides statistics about the Detokenization and Clean_table operations.

We observe that the Detokenization operation (Figure 5e) for a table filled with tokens takes on average 660 seconds, i.e., 11 minutes, with a very low standard deviation of 26 seconds. This represents only $6 \mu$ s for a single detokenization, which is far less than the expected $100 \mathrm{~ms}$ timeframe.

The Clean_table operation was also run on a table filled with depleted tokens. The median time for cleaning the table (Figure $5 \mathrm{f}$ ) is 36.6 seconds with a standard derivation of 0.43 seconds. This illustrates that our upcycling tokenization technique can cope well with large data as it is able to clean the table in a very short time.

\subsection{RAM Usage}

The theoretical amount of RAM usage depends on the row length and encryption design of the table. Depending on the security requirements, one can decide whether to encrypt the table or not. If it is the case, the table would be encrypted with Advanced Encryption Standard (AES) and thus in 128-bit blocks.

For our experiments ( 64 bits expiry and a 64 bit auth), the data in a row being stored on 232 bits, the encryption would be done on 2 blocks of 128 bits. With $n_{\max }=10^{8}$ rows to store, 25.6GB of RAM are necessary for the storage of the encrypted table. If we choose not to encrypt the table, only 23.2 GB of RAM are used for storage. Note that if auth is bigger, extra AES blocks must be added which would increase the memory requirements for the table.

\section{CONCLUSIONS}

In this paper, we proposed a solution for tokenization systems for Credit card numbers. This system is based on the possibility to keep a full table of tokens in RAM so that computations are fast enough to guarantee a tokenization within a $100 \mathrm{~ms}$ timeframe as long as the table is not filled more than $99.87 \%$. An external database allows us to keep transaction data for auditability while also allowing the table to be cleaned regularly from expired tokens.

Our approach is still limited by its single point of failure nature and extra mechanisms should be added to improve availability and resilience.

For example, each modification in the RAM could be duplicated in a SSD, in order to have a non-volatile backup almost up to date. The RAM can also be duplicated into another RAM to increase availability with minimal losses in case of failure. The TSP role could also be split across multiple computers by allocating a partition of the token space to each computer.

Also, in order to reduce the memory requirements of the table and store more data per row, one could implement a scalable data structure such as a binary tree or more advanced dynamic storage mechanisms.

Our experiments showed that our implementation satisfies the specifications of the Token Service Providers, and is efficient enough to manage 6 times the current number of worldwide credit card transactions.

\section{ACKNOWLEDGEMENTS}

This work was done in cooperation with the BE YS GROUP through its Pay and Research branches in REDOCS 2020 based on the BE YS PAY TSP project. The authors would also like to thank Pascal Lafourcade and Olivier Blazy for their support, as well as Marius Lombard-Platet for his insights. 


\section{REFERENCES}

Albarel., D. L. (2021). Tokenization. https://github.com/ DianeLeblancAlbarel/Tokenisation

Bellare, M., Ristenpart, T., Rogaway, P., and Stegers, T. (2009). Format-Preserving Encryption. In Jacobson, M. J., Rijmen, V., and Safavi-Naini, R., editors, Selected Areas in Cryptography, pages 295-312. Springer Berlin Heidelberg.

Cachin, C., Camenisch, J., Freire-Stögbuchner, E., and Lehmann, A. (2017). Updatable Tokenization: Formal Definitions and Provably Secure Constructions. In Kiayias, A., editor, Financial Cryptography and Data Security, pages 59-75. Springer International Publishing.

CardRates (2020). The Average Number of Credit Card Transactions Per Day \& Year. https://www.cardrates.com/advice/number-of-creditcard-transactions-per-day-year/

Durak, F. B. and Vaudenay, S. (2017). Breaking the FF3 Format-Preserving Encryption Standard over Small Domains. In Katz, J. and Shacham, H., editors, $A d$ vances in Cryptology - CRYPTO 2017, pages 679707, Cham. Springer International Publishing.

Díaz-Santiago, S., Rodriguez-Henriquez, L. M., and Chakraborty, D. (2014). A cryptographic study of tokenization systems. In 11th International Conference on Security and Cryptography (SECRYPT), pages 16.

Halevi, S. and Krawczyk, H. (1999). Public-key cryptography and password protocols. ACM Trans. Inf. Syst. Secur., 2(3):230-268.

Hoang, V. T., Tessaro, S., and Trieu, N. (2018). The Curse of Small Domains: New Attacks on Format-Preserving Encryption. In Shacham, H. and Boldyreva, A., editors, Advances in Cryptology CRYPTO 2018, pages 221-251, Cham. Springer International Publishing.

International Organization for Standardization (2017). ISO/IEC 7812-1:2017 Identification cards - Identification of issuers - Part 1: Numbering system. Technical report, International Organization for Standardization. https://www.iso.org/obp/ui/\#iso:std:iso-iec: 7812:-1:ed-5:v1:en

Johnson, D., Menezes, A., and Vanstone, S. (2001). The elliptic curve digital signature algorithm (ecdsa). $1(1): 36-63$.

Krebs, Brian (2019). A Month After 2 Million Customer Cards Sold Online, Buca di Beppo Parent Admits Breach. https://krebsonsecurity.com/tag/davincibreach/

Liu, Z., Jia, C., Li, J., and Cheng, X. (2010). Formatpreserving encryption for datetime. In 2010 IEEE International Conference on Intelligent Computing and Intelligent Systems, volume 2, pages 201-205. IEEE.

Luhn, H. P. (1960). Computer For Verifying Numbers. US2950048A.

N. Owen, W. and Shoemaker, E. (2008). Multi-factor authentication system.
NIST (2020). Methods for Format-Preserving Encryption: NIST Requests Public Comments on Draft Special Publication 800-38G Revision 1. https: //www.nist.gov/news-events/news/2019/02/methodsformat-preserving-encryption-nist-requests-publiccomments-draft

Payment Card Industry (2015). Tokenization Product Security Guidelines - Irreversible and Reversible Tokens. https://www.pcisecuritystandards.org/documents/ Tokenization_Product_Security_Guidelines.pdf

Payment Card Industry (2020). Payment Card Industry Security Standards. https://www.pcisecuritystandards. org/pci_security/maintaining_payment_security

Sussman, B. (2020). 'BIGBADABOOM!' Carding Forum Selling Millions of Records from Wawa Stores Data Breach. https://www.secureworldexpo.com/industrynews/carding-forum-wawa-data-breach-update

Thales group (2018). Card Data from 5M Customers Stolen in Data Breach at Saks Fifth Avenue, Lord \& Taylor. https://dis-blog.thalesgroup.com/security/2018/ 04/03/saksfifthavenuedatabreach/

U.S. Department of Commerce (2020). Quarterly Retail Ecommerce Sales. https://www.census.gov/retail/mrts/ www/data/pdf/ec_current.pdf

Voltage Security (2012). Voltage secure stateless tokenization. https://www.voltage.com/wpcontent/uploads/Voltage_White_Paper_SecureData_ SST_Data_Protection_and_PCI_Scope_Reduction_ for_Todays_Businesses.pdf

Whatman, P. (2020). Credit card statistics 2020: 65+ facts for Europe, UK, and US. https://blog.spendesk.com/ en/credit-card-statistics- 2020 